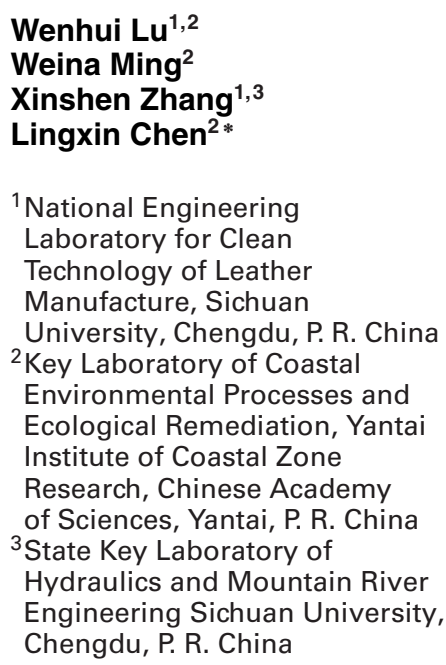

Received March 13, 2016

Revised May 20, 2016

Accepted July 13, 2016
Research Article

\section{Molecularly imprinted polymers for dispersive solid-phase extraction of phenolic compounds in aqueous samples coupled with capillary electrophoresis}

\begin{abstract}
A simple and sensitive method for the simultaneous determination of seven phenolic compounds in aqueous samples was developed by dispersive solid-phase extraction (DSPE) coupled with capillary electrophoresis (CE), namely phenol, 4-chlorophenol (4-CP), pentachlorophenol (PCP), 2,4,6-trichlorophenol (2,4,6-TCP), 2,4-dichlorophenol (2,4-DCP), 2-chlorophenol (2-CP) and 2,6-dichlorophenol (2,6-DCP). Molecularly imprinted polymers (MIPs) prepared by precipitation polymerization with 2,4,6-trichlorophenol (2,4,6-TCP) as template were used as DSPE sorbents to selectively adsorb the phenols. Various parameters affecting the extraction efficiency were evaluated and excellent CE separation was achieved within $13.5 \mathrm{~min}$ by using $15 \mathrm{mmol} / \mathrm{L}$ borate buffer containing 15\% (v/v) methanol at $\mathrm{pH}=9.8$. Under the optimized conditions, good linearity was obtained in the range of 2-200 $\mu \mathrm{g} / \mathrm{L}$ for phenol, 2,4-DCP and 2,6-DCP, 2-300 $\mu \mathrm{g} / \mathrm{L}$ for 4-CP and 2-CP, 1.5-150 $\mu \mathrm{g} / \mathrm{L}$ for PCP, and 2-400 $\mu \mathrm{g} / \mathrm{L}$ for 2,4,6-TCP, with the correlation coefficient $\left(R^{2}\right)$ higher than 0.9938 . Limits of detection and limits of quantification were in the range of $0.18-0.44 \mu \mathrm{g} / \mathrm{L}$ and $0.59-1.45 \mu \mathrm{g} / \mathrm{L}$, respectively. The developed MIPs-DSPECE method was also successfully applied to determine the seven phenolic compounds in lake, tap, seawater and tannery wastewater. The average recoveries at three different spiked levels ranged from 70.75 to $106.7 \%$ with the relative standard deviations of $1.15-6.28 \%$.
\end{abstract}

\section{Keywords:}

Aqueous samples / Capillary electrophoresis / Dispersive solid-phase extraction / Molecularly imprinted polymers / Phenolic compounds

DOI 10.1002/elps.201600119

Additional supporting information may be found in the online version of this article at the publisher's web-site

\section{Introduction}

Phenolic compounds are generated in the production of pesticides, antioxidants, plastics, drugs, dyes, paper, textile and leather, and have widely existed in environmental water and soil at concentrations below $\mu \mathrm{g} / \mathrm{L}$ level [1]. In general, phenol is the degradation product of lignin used in the paper industry, and chlorophenols with strongly pungent odor can be generated from phenol in the chlorinating of drinking water and the degradation of phenoxy herbicides [2]. These phenolic compounds can be released into environment directly

Correspondence: Professor Xinshen Zhang, National Engineering Laboratory for Clean Technology of Leather Manufacture, and State Key Laboratory of Hydraulics and Mountain River Engineering, Sichuan University, Chengdu 610065, P. R. China

E-mail: zhangxinshen@126.com or indirectly by industrial effluents, by-products from natural and synthetic chemicals, which can seriously affect human health and environmental quality [3]. Because of their high toxicity, unpleasant organoleptic properties, poor biodegradability and potential carcinogenicity, several phenolic compounds have been listed as priority pollutants by United State Environmental Protection Agent [4]. Therefore, developing reliable, sensitive and efficient analytical methods is very essential for the determination of trace level phenolic compounds.

Currently, gas chromatography (GC) [5], high performance liquid phase chromatography (HPLC) [6] and capillary electrophoresis (CE) [7] have been widely applied for the

\footnotetext{
*Additional corresponding author: Professor Lingxin Chen E-mail: lxchen@yic.ac.cn

Colour Online: See the article online to view Figs. 1 and 2 in colour.
} 
analysis of phenolic compounds. They are usually needed to derive with suitable derivatization reagent to increase their volatility before injection into GC. HPLC coupled with various detectors are used for the determination of phenols, such as ultraviolet (UV) [8-10], fluorescence [11] and mass spectroscopy (MS) [12]. To improve the sensitivity of CE, online/inline stacking techniques have been presented, such as large volume sample stacking [13]. However, these detection techniques are difficult to achieve the limits of detection (LODs) and limits of quantitation (LOQs) required for the direct determination of phenols in complicated matrices without sample pretreatment. Many traditional and novel pretreatment techniques have been developed to enrich phenols in different matrices, such as liquid-liquid extraction, liquidphase microextraction [14], dispersive liquid-liquid microextraction (DLLME) [15], solid-phase extraction (SPE) [16], solidphase microextraction (SPME) [17-19] and stir-bar sorptive extraction [20]. For instance, Liu et al. used DLLME to offline pretreat and online LVSS and sweeping coupled with micellar electrokinetic chromatography (MEKC) for the determination of phenols in water samples [21]. LODs and LOQs for six phenols were in the range of $0.2-1.0 \mu \mathrm{g} / \mathrm{L}$ and $0.6-3.4 \mu \mathrm{g} / \mathrm{L}$, respectively. To achieve high enrichment factor and detection sensitivity, Verma et al. developed SPE combined with headspace single-drop microextraction (HS-SDME) coupled with HPLC for the determination of chlorophenols in water samples [22]. When using coupled SPE and HS-SDME, the obtained LODs for chlorophenols in the range of 0.04$0.08 \mu \mathrm{g} / \mathrm{L}$ were lower than using HS-SDME alone with the range of $1.5-3.9 \mu \mathrm{g} / \mathrm{L}$. Abolghasemi and Yousefi presented SPME based on high temperature three dimensionally honeycomb layered double hydroxide material coupled with GCMS for the analysis of some phenolic and polycyclic aromatic hydrocarbon compounds in aqueous sample solutions [23]. LODs were ranging from 0.02 to $5.8 \mu \mathrm{g} / \mathrm{L}$. SPE as a wellestablished pretreatment technique has been widely applied in environmental, food and biological fields. Separation on the most commonly used SPE sorbents, such as $\mathrm{C}_{18}, \mathrm{C}_{8}$, florisil and $\mathrm{Al}_{2} \mathrm{O}_{3}$, is based on physicochemical retention on the functionalized surface [24]. However, besides target analytes, such SPE cartridge can also retain other matrix compounds. Therefore, many studies focus on the development of SPE sorbents, aim to synthesize high selectivity, good adsorptive performance and chemical or mechanical stability materials [25].

Molecularly imprinted polymers (MIPs) are materials synthesized in the presence of template for the formation of artificially generated template-complementary recognition sites which can specifically rebind template molecule and the structural analogue [26]. Because of the high selectivity and good adsorption capacity of MIPs, they have been extensively utilized as selective SPE sorbent materials [27-29]. Recently, several studies have been reported on using MIPs as SPE sorbents for adsorption of phenols [30-32]. For example, Lin et al. prepared 2,4,6-TCP MIPs by bulk polymerization and applied to SPE for selective preconcentration of phenolic compounds in environmental water samples [31]. The obtained polymers should be crushed, ground and sieved before usage, with irregular shape and heterogeneous size distribution. To prepare regular MIPs beads or microspheres with suitable physical characteristics (size, porosity and surface area), several polymerization methods have been proposed, such as suspension polymerization, multi-step swelling and precipitation polymerization. The preparation procedure of precipitation polymerization is similar to bulk polymerization but with the addition of larger amounts of porogens, and is considered simple and easy.

Herein, we prepared MIPs by precipitation polymerization using 2,4,6-TCP as template molecule, methacrylic acid (MAA) as a functional monomer and ethylene glycol dimethacrylate (EGDMA) as a cross-linker. The morphology, adsorption performance and selectivity of MIPs were estimated. Dispersive solid-phase extraction (DSPE), firstly developed by Anastassiades in 2003 [33], is based on the SPE methodology and the sorbents are directly added into the sample solution without conditioning. In this study, we attempted to use 2,4,6-TCP MIPs as DSPE sorbents coupled with CE for the simultaneous separation of seven phenolic compounds in water samples. The main parameters affecting DSPE efficiency were investigated systematically, such as amount of MIPs, sample $\mathrm{pH}$, extraction time, desorption solvent and time. Under the optimized conditions, the established MIPs-DSPE-CE method showed high sensitivity and selectivity which could be potentially applied to determine phenols in complicated samples.

\section{Materials and methods}

\subsection{Reagents and materials}

For CE separation, HPLC grade reagents of phenol, 2-chlorophenol (2-CP), 4-chlorophenol (4-CP), 2,4-dichlorophenol (2,4-DCP), 2,6-dichlorophenol (2,6-DCP) and 2,4,6-trichlorophenol (2,4,6-TCP) were obtained from Sigma-Aldrich (Shanghai, China) and the standard stock solutions were prepared by dissolving them in methanol of a concentration of $1000 \mathrm{mg} / \mathrm{L}$. The standard solution of PCP in methanol with concentration of $1000 \mathrm{mg} / \mathrm{L}$ was purchased from Aladdin (Shanghai, China). All the standard solutions were stored at $4^{\circ} \mathrm{C}$ in a refrigerator. Sodium tetraborate decahydrate $\left(\mathrm{Na}_{2} \mathrm{~B}_{4} \mathrm{O}_{7} \cdot 10 \mathrm{H}_{2} \mathrm{O}\right)$, sodium dihydrogen phosphate $\left(\mathrm{NaH}_{2} \mathrm{PO}_{4}\right)$, disodium hydrogen phosphate $\left(\mathrm{Na}_{2} \mathrm{HPO}_{4}\right)$ were supplied from Sinopharm Chemical Reagent Co., Ltd. (Shanghai, China).

For polymer synthesis, MAA and EGDMA were purchased from Sigma-Aldrich and purified prior to use in order to remove stabilizers. 2,2'-azo-bis-isobutyronitrile (AIBN) was attained from Sinopharm Chemical Reagent Co., Ltd. (Shanghai, China) and recrystallized in ethanol prior to use. Acetonitrile, methanol and acetic acid were obtained by Sinopharm Chemical Reagent Co., Ltd. (Shanghai, China).

Ultrapure water with the specific resistances of 18.2 $\mathrm{M} \Omega \cdot \mathrm{cm}$ was produced by Pall cascada $^{\mathrm{TM}}$ lab water 
purification system (Pall Corp., USA) for aqueous solution preparation throughout the study. All other solvents and chemicals were of analytical grade and used directly without further purification.

\subsection{Instrumentation}

CE measurements were carried out on a Beckman P/ACE ${ }^{\mathrm{TM}}$ MDQ Capillary Electrophoresis System (USA) equipped with a diode array detector (DAD). The detection wavelength was set at $195 \mathrm{~nm}$ for phenol, 4-CP and 2-CP, $214 \mathrm{~nm}$ for 2,4, 6-TCP, 2,4-DCP and 2,6-DCP, and $228 \mathrm{~nm}$ for PCP. The capillary temperature was maintained at $25^{\circ} \mathrm{C}$ and the applied voltage was $+15 \mathrm{kV}$ and pressure injection was performed using 0.5 psi for $5 \mathrm{~s}(1 \mathrm{psi}=6894.76 \mathrm{~Pa})$. Bare fused-silica capillary with $50.2 \mathrm{~cm}$ total length, $40 \mathrm{~cm}$ effective length, and $75 \mu \mathrm{m}$ i.d. was used as a separation column (Yongnian Optical Fiber Co. Ltd., Hebei, China). The pH value measurements were made with an Rex pH meter (Shanghai Precision Scientific Instrument Corporation, Shanghai, China). New capillary was preconditioned by rinsing with methanol for $5 \mathrm{~min}$, water for $5 \mathrm{~min}, 1 \mathrm{~mol} / \mathrm{L} \mathrm{NaOH}$ for $30 \mathrm{~min}$, water for $10 \mathrm{~min}$ and running buffer for $30 \mathrm{~min}$. Each day, the capillary was conditioned by flushing with water for $5 \mathrm{~min}, 1 \mathrm{~mol} / \mathrm{L}$ $\mathrm{NaOH}$ for $5 \mathrm{~min}$ and then water for $5 \mathrm{~min}$. Between each run, the capillary was rinsed with separation buffer for $5 \mathrm{~min}$, which consisted of $15 \mathrm{mmol} / \mathrm{L}$ borate buffer containing 15\% (v/v) methanol $(\mathrm{pH}=9.8)$. All electrolytes and samples were filtered through $0.22 \mu \mathrm{m}$ membrane filters and degassed by ultrasonic prior to analysis.

The phase separation was conducted with centrifuge (Xiangyi Centrifuge Instrument Co. Ltd., Hunan, China). The ultrasonic process was carried out using KQ2200B ultrasonic device at a frequency of $40 \mathrm{kHz}$ with an ultrasound input power of $100 \mathrm{~W}$ (Kunshan Ultrasonic Instrument Co. Ltd., Jiangsu, China). Scanning electron microscope (SEM, Hitachi S-4800, Japan) was used for morphological evaluation. All samples were sputter-coated with gold before SEM analysis. Fourier transform infrared (FT-IR) spectrometer (Thermo Nicolet Corporation, USA) was employed to examine the infrared spectra of sample using a pressed $\mathrm{KBr}$ tablet. UV-vis absorption spectra were measured on a Thermo Scientific NanoDrop 2000/2000C spectrophotometer (USA).

\subsection{Preparation of 2,4,6-TCP MIPs}

The MIPs were prepared by precipitation polymerization according to noncovalent approach and the schematic procedure was shown in Fig. 1A. The template molecule 2,4,6-TCP $(0.1 \mathrm{mmol})$ was dissolved in $20 \mathrm{~mL}$ acetonitrile in a $50 \mathrm{~mL}$ glass flask and $0.4 \mathrm{mmol}$ MAA (monomer) was added to the solution. After the $12 \mathrm{~h}$ pre-polymerization process at $4^{\circ} \mathrm{C}, 2 \mathrm{mmol}$ EGDMA (cross-linker) and $10 \mathrm{mg}$ AIBN (initiator) were added to the mixture solution. Then, the above

A

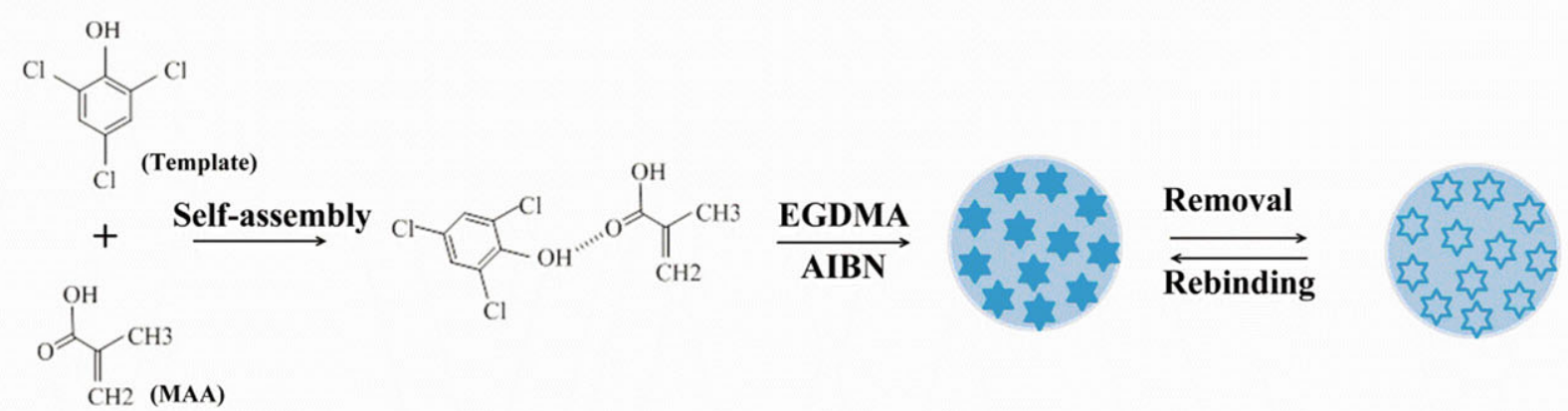

B

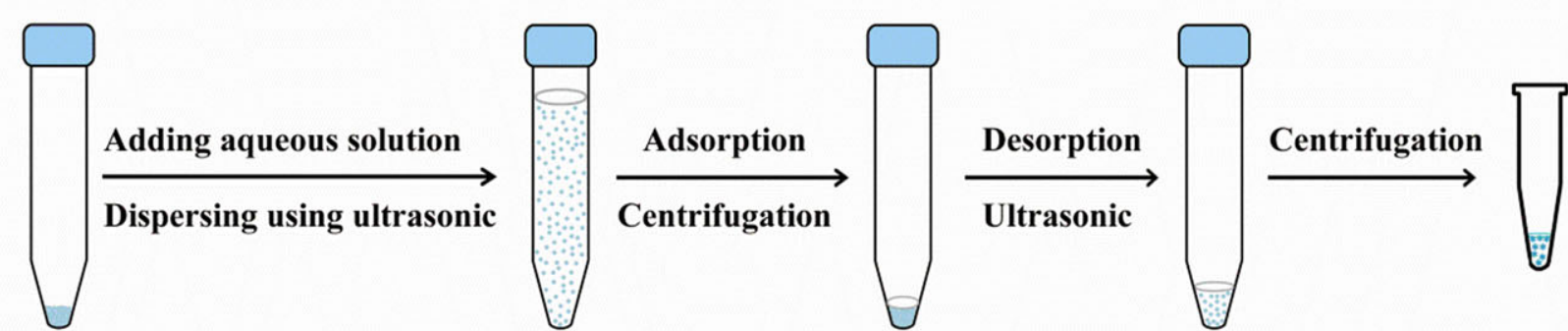

Template

MIPs

Figure 1. Schematic procedures of the preparation procedure of MIPs by precipitation polymerization (A) and the obtained MIPs used for DSPE (B). 
solution was deoxygenated with nitrogen for $15 \mathrm{~min}$ to remove dissolved oxygen and the flask was sealed under a nitrogen atmosphere and placed in water bath. The temperature of water bath ramped from room temperature to $60^{\circ} \mathrm{C}$ around $2 \mathrm{~h}$ and kept at this temperature for $24 \mathrm{~h}$ to polymerize. After the polymerization, the resultant polymers were washed with acetonitrile twice to remove the unreacted reagents and then washed with acetonitrile/acetic acid $(90: 10, v / v)$ to remove the template molecules until no template was detected by UV-vis. Then, the obtained MIPs were dried in a vacuum drying oven at $40^{\circ} \mathrm{C}$ for use. As a control, the corresponding non-imprinted polymers (NIPs) were also synthesized using the same procedure only without the template 2,4,6-TCP.

\subsection{Characterization of MIPs}

The morphological evaluation of MIPs and NIPs was performed by SEM and all samples were sputter-coated with gold before analysis. To investigate the adsorption capacity of polymers to 2,4,6-TCP, $2 \mathrm{mg}$ MIPs or NIPs were added into $2 \mathrm{~mL}$ aqueous solution of 2,4,6-TCP with various concentrations in $4 \mathrm{~mL}$ centrifuge tubes. Then the suspensions were sealed and shaken for $24 \mathrm{~h}$ at room temperature to ensure the equilibrium. After filtration with $0.22 \mu \mathrm{m}$ cellulose acetate membrane (MCM) filters, the solution containing the free 2,4,6-TCP was measured by UV-vis. The adsorption capacity $(Q)$ was calculated according to the following equation:

$Q=\left(C_{0}-C_{1}\right) \times V / m$.

In this equation, $C_{0}$ and $C_{1}$ are the concentration of 2,4, 6-TCP in solution before and after adsorption, $V$ is the volume of the solution, and $m$ is the mass of MIPs or NIPs.

\subsection{MIPs-DSPE procedure and sample preparation}

The obtained MIPs were used as sorbents for DSPE and the procedure was schematically shown in Fig. 1B. $10 \mathrm{mg}$ MIPs sorbents and $10 \mathrm{~mL}$ aqueous solution containing suitable concentration of standard solution were added into a conical centrifugation tube. The sorbents were dispersed into the solution by ultrasonic about $1 \mathrm{~min}$ and formed suspension solution, followed by centrifugation at $9000 \mathrm{rpm}$ for $10 \mathrm{~min}$. After removing the supernatant as clean as possible and drying the sorbents by vacuum drying oven, $30 \mu \mathrm{L}$ acetonitrile/acetic acid $(90: 10, \mathrm{v} / \mathrm{v})$ was added into the tube to elute the analytes by ultrasonic $4 \mathrm{~min}$, then centrifugation was carried out at $9000 \mathrm{rpm}$ for $10 \mathrm{~min}$ to collect the supernatant for CE analysis.

Lake water was collected from an artificial lake located in Yantai city (China), natural seawater was collected from Yantai city (China) and tannery wastewater was collected from our laboratory, and all these samples were stored at $4^{\circ} \mathrm{C}$ in a refrigerator before analysis. Tap water was collected after it had flowed for about $5 \mathrm{~min}$ in the laboratory when needed.
Before use, the samples were filtered through a $0.22 \mu \mathrm{m}$ filter to remove suspended particles prior to extraction.

\section{Results and discussion}

\subsection{Characterization of MIPs}

The purpose of the present study was to prepare MIPs sorbents by precipitation polymerization which can selectively adsorb phenols in water samples. SEM images of 2,4,6TCP MIPs and NIPs were shown in Supporting Information Fig. S1. Under the optimum preparation conditions, MAA was chosen as functional monomer because the hydrogen bond can be formed between carboxyl acid group of MAA and phenolic hydroxyl group of 2,4,6-TCP, along with acetonitrile as a porogen, EGDMA as a cross-linker and AIBN as an initiator. From the figure, we can see that the obtained MIPs and NIPs were spherical particles with relatively uniform size, and the particle size of MIPs was slightly larger than NIPs because template can influence the nucleation stage of precipitation polymerization.

FT-IR spectra of MIPs before and after template molecule (2,4,6-TCP) removal were shown in Supporting Information Fig. S2. Form curves a and b, strong absorption bands at around 1732 and $3429 \mathrm{~cm}^{-1}$ were observed, which could be attributed to the stretching vibration of $\mathrm{C}=\mathrm{O}$ and $\mathrm{O}-$ $\mathrm{H}$ from carboxylic acid of MAA. Symmetric and asymmetric stretching vibration of C-O from EGDMA were found at 1257 and $1149 \mathrm{~cm}^{-1}$, respectively [34]. The peaks at 2989 and $2958 \mathrm{~cm}^{-1}$ indicated the presence of $\mathrm{C}-\mathrm{H}$ stretching bands of both $-\mathrm{CH}_{3}$ and $-\mathrm{CH}_{2}$. As seen from curve $\mathrm{b}$, the disappearance of $\mathrm{C}-\mathrm{Cl}$ bond stretching vibration at $732 \mathrm{~cm}^{-1}$ and $\mathrm{C}=\mathrm{C}$ stretching vibration in benzene ring at 1570 and $1469 \mathrm{~cm}^{-1}$ could demonstrate the successful imprinting of 2,4,6-TCP $[35,36]$.

The binding experiments were carried out as described in Section 2.4 and the binding isotherms were obtained with 2,4,6-TCP concentration from 80 to $600 \mathrm{mg} / \mathrm{L}$ as shown in Supporting Information Fig. S3A. Since the determination of phenols was applied in aqueous samples, the binding assays were accomplished in water solutions. As seen from the figure, the adsorption capacity of 2,4,6-TCP increased fast with the increase of initial concentration; at equilibrium concentrations higher than $500 \mathrm{mg} / \mathrm{L}$, adsorption of MIPs became stable. NIPs had the same trend as MIPs but with lower adsorption capacity. That is because of the adsorption-desorption equilibrium between polymers and template molecule. When the initiate concentration of 2,4,6-TCP was low, the adsorbed template only occupied a small number of recognition sites. When continuously increasing the concentration, more and more recognition sites were occupied and even saturated, and accordingly the curves began to level off. The results showed that MIPs had higher adsorption capacity to 2,4,6-TCP than NIPs, and the maximum adsorption capacity was estimated to $543 \mathrm{mg} / \mathrm{g}$ for MIPs and $290 \mathrm{mg} / \mathrm{g}$ for NIPs; such high adsorption ability 
would greatly facilitate extracting and enriching trace level of 2,4,6-TCP in water samples. For further evaluation the selectivity of MIPs, the binding capacities of phenol, 2-CP, 4-CP, 2,4-DCP, 2,6-DCP and PCP were also investigated. As seen from Supporting Information Fig. S3B, the MIPs also showed certain binding capacities of other structurally related compounds. In contrast, NIPs gave lower binding capacities than MIPs for seven phenolic compounds because of no specific binding sites in NIPs. It also demonstrated the potential of MIPs to selectively adsorb these phenolic compounds in water samples.

\subsection{Condition optimization of MIPs-DSPE procedure}

According to the above characterization of MIPs, we proposed to use the 2,4,6-TCP MIPs as sorbents of DSPE coupled with CE-DAD for enrichment and determination of seven phenols in aqueous samples. In order to obtain the maximum extraction efficiency, the MIPs-DSPE procedure was optimized by evaluating the amount of MIPs, sample $\mathrm{pH}$, extraction time, desorption solvent and time.
Compared with the commercial SPE sorbents with particle sizes of 40-60 $\mu \mathrm{m}$, the obtained MIPs have more uniform and smaller particle size $(\sim 1 \mu \mathrm{m})$. Therefore, these MIPs sorbents are more suitable for DSPE and satisfactory results may be obtained by using less amount of sorbent. The effect of sorbent dosage was investigated by using 5-15 mg MIPs for $10 \mathrm{~mL}$ sample solution containing $100 \mu \mathrm{g} / \mathrm{L}$ seven phenols and the mixture was treated using the method of Section 2.5. As shown in Fig. 2A, the extraction efficiency increased from 5 to $10 \mathrm{mg}$ and remained almost constant up to $15 \mathrm{mg}$. The results suggested that $10 \mathrm{mg}$ MIPs were sufficient for extraction of the phenols, and thus were chosen for the further study.

Because of acid or base properties of phenolic compounds, sample $\mathrm{pH}$ can influence the form of phenols and their water solubility. A series of assays were operated to optimize sample $\mathrm{pH}$ from 2 to 9 by adding varied amounts of $\mathrm{HCl}$ or $\mathrm{NaOH}$. Results showed that no obvious change of extraction efficiency was obtained in the range of $\mathrm{pH} 2-$ 9 (Fig. 2B). The possible reason is that most of the phenolic compounds are existent in the molecule form related to $\mathrm{pKa}$ values (i.e., 10.09 for phenol, 8.52 for 2-CP, 9.43
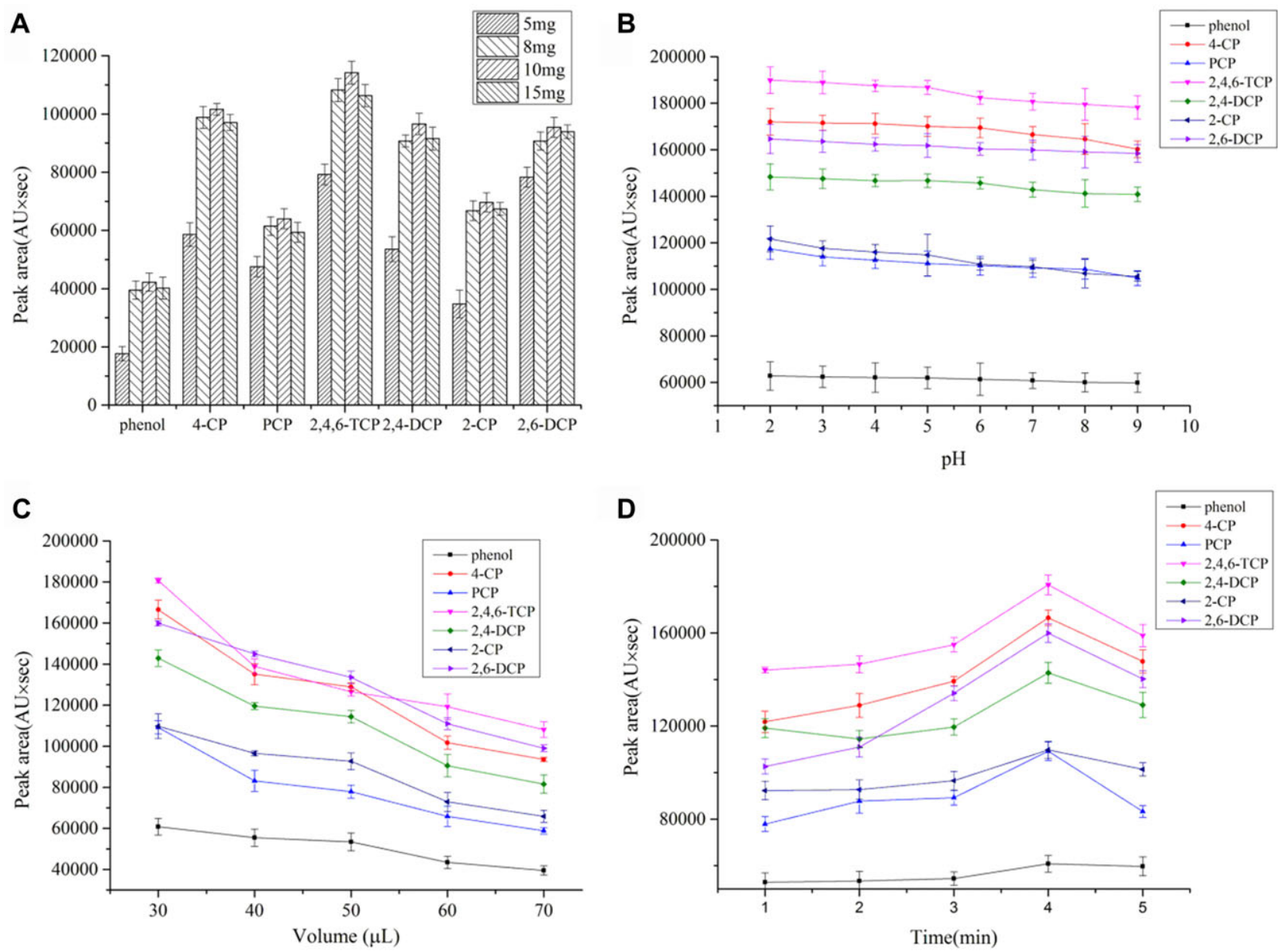

Figure 2. Optimization of MIPs-DSPE procedure. Effects of (A) amount of MIPs, (B) sample pH, (C) volume of desorption solvent and (D) desorption time. 
for 4-CP, 7.89 for 2,4-DCP, 7.42 for 2,4,6-TCP, 6.79 for 2, 6-DCP and 4.93 for PCP) [2, 15, 37]. Therefore, neutral condition for sample $\mathrm{pH}$ was selected in order to simplify the experiment.

In the process of DSPE, it is necessary to allow the sorbents to be dispersed appropriately into the sample solution, and thereby provide sufficient contact area and transfer time for the analytes and MIPs to reach the adsorption equilibrium. In this experiment, MIPs and sample solution were added into the tube subsequently and the MIPs were completely dispersed into the solution by ultrasonic. Extraction time was defined as the time of oscillation after the formation of suspension solution by ultrasonic. The effect of extraction time was investigated by changing the oscillation time from 0 to $3 \mathrm{~h}$. According to the results, no significant change occurred as the extraction time increasing. This phenomenon can be attributed to the easy dispersibility of MIPs in aqueous solution.

The type of desorption solvents can influence the desorption efficiency of analytes from MIPs sorbents. Different kinds of solvents including methanol, acetonitrile, acetonitrile/acetic acid (at the volume ratio of 90:10, 85:15) were investigated. Acetonitrile exhibited better desorption efficiency than methanol, which may be the same as the polymerization solvent [38]. The adding of acetic acid in acetonitrile can increase the extraction efficiency of 2,4,6-TCP, but more acid can result in low desorption efficiency. The possible reason is that the carboxyl of acetic acid can form hydrogen bond with 2,4,6-TCP, which competes with the functional group in binding sites of polymers. However, the hydrogen bond may become weak due to the addition of more acetic acid, and hence influence desorption. As a result, acetonitrile containing $10 \%(\mathrm{v} / \mathrm{v})$ acetic acid was chosen in the following study. Besides, the volume of desorption solvent is also an important parameter that effectively influences the extraction efficiency. As shown in Fig. 2C, the extraction efficiency decreased with the volume increasing from 30-70 $\mu \mathrm{L}$; the maximum efficiencies of seven phenolic compounds were achieved when using $30 \mu \mathrm{L}$. This means that $30 \mu \mathrm{L}$ is enough to elute all the target analytes, and the desorption is very likely to reach an equilibrium. Accordingly, with the volume continuously increasing, the extraction efficiency decreased. On the other hand, when using less volume of desorption solvent like $20 \mu \mathrm{L}$, the analytes couldn't be desorbed sufficiently from MIPs, presenting low peak area and poor reproducibility (data not shown). Therefore, to obtain the reproducible desorption and the highest extraction efficiency, as well as to ensure the desorption solvent sufficiently get in touch with the sorbents and easily handled with the centrifugation, $30 \mu \mathrm{L}$ acetonitrile/acetic acid $(90: 10, \mathrm{v} / \mathrm{v})$ was employed as the desorption solution.

To completely desorb the analytes from MIPs, desorption time was also estimated in the range of 1-5 min. Results of investigation on desorption time were shown in Fig. 2D. As seen from the figure, the optimum extraction efficiencies were achieved at $4 \mathrm{~min}$ for all the seven phenolic compounds. For phenol, the extraction efficiency increased slowly in the first $3 \mathrm{~min}$ and then remained stable from 4 to $5 \mathrm{~min}$. For the other six phenolic compounds, the extraction efficiencies increased obviously from 1 to $4 \mathrm{~min}$, and then decreased with time increasing to $5 \mathrm{~min}$. So, $4 \mathrm{~min}$ as desorption time was sufficient for the complete removal of seven phenols from MIPs.

\subsection{Method performance}

Under the above-optimized MIPs-DSPE conditions, the typical electropherograms of standard solutions of seven phenols were shown in Fig. 3. Then, the analytical performance of this developed MIPs-DSPE-CE method was investigated, and the results were presented in Table 1 . The working solutions containing seven phenols at various concentrations were analyzed to establish the calibration curves. As listed in the table, good linearity was attained in the range of 2-200 $\mu \mathrm{g} / \mathrm{L}$ for phenol, 2,4-DCP and 2,6-DCP, 2$300 \mu \mathrm{g} / \mathrm{L}$ for $4-\mathrm{CP}$ and $2-\mathrm{CP}, 1.5-150 \mu \mathrm{g} / \mathrm{L}$ for PCP, and $2-400 \mu \mathrm{g} / \mathrm{L}$ for $2,4,6-\mathrm{TCP}$, with correlation coefficient $\left(R^{2}\right)$ higher than 0.9938 . The LODs were obtained based on the peak height as three times of background noise $(\mathrm{S} / \mathrm{N}=3)$, in the range of $0.18-0.44 \mu \mathrm{g} / \mathrm{L}$. The LOQs calculated based on the peak height being ten times the background noise $(\mathrm{S} / \mathrm{N}=10)$ were in the range of $0.59-1.45 \mu \mathrm{g} / \mathrm{L}$. The relative standard deviations (RSD) at $100 \mu \mathrm{g} / \mathrm{L}$ of all analytes $(n=5)$ for the intraday and interday precisions ranged from 1.91-6.04\% and 2.02-6.79\%, respectively. The enrichment factor (EF) was defined as the ratio between the concentration of analyte before and after MIPs-DSPE. The calculated EFs within 70-242 showed relatively high pretreatment efficiency.

Comparisons of the analytical performance of the proposed MIPs-DSPE-CE method with other MIPs based methods for phenols in different matrices were shown in Supporting Information Table S1. As seen, the CE analysis of seven phenols needs shorter time ( 13 min) than HPLC (16$20 \mathrm{~min})$ [30, 31], MEKC ( $35 \mathrm{~min})[32]$ and GC ( $20 \mathrm{~min})$ [39]. For DSPE procedure, MIPs polymers are completely dispersed in sample solution, which increases the contact opportunity of target analytes and shortens the extraction time. Also, as can be seen from the table, there are various polymerization methods used for the preparation of MIPs for selective adsorption of phenols. Although bulk polymerization uses less porogen than precipitation polymerization, the obtained bulk polymers should be crushed, ground and sieved before used to the pretreatment process [31,39]. Magnetic MIPs can be easily separated from sample solution using an external magnetic field without additional centrifugation or filtration, which simplifies the extraction procedure [32], but the MEKC analysis needs longer time to separate three chlorophenols than our method. The obtained LODs were lower than that of the HPLC [30,31] or comparable to that of the GC [39] methods, except the method of online LVSS coupled with MEKC [32]. Nevertheless, the benefits of MIPs-DSPE-CE method are still obvious, like high sensitivity and selectivity, simple 

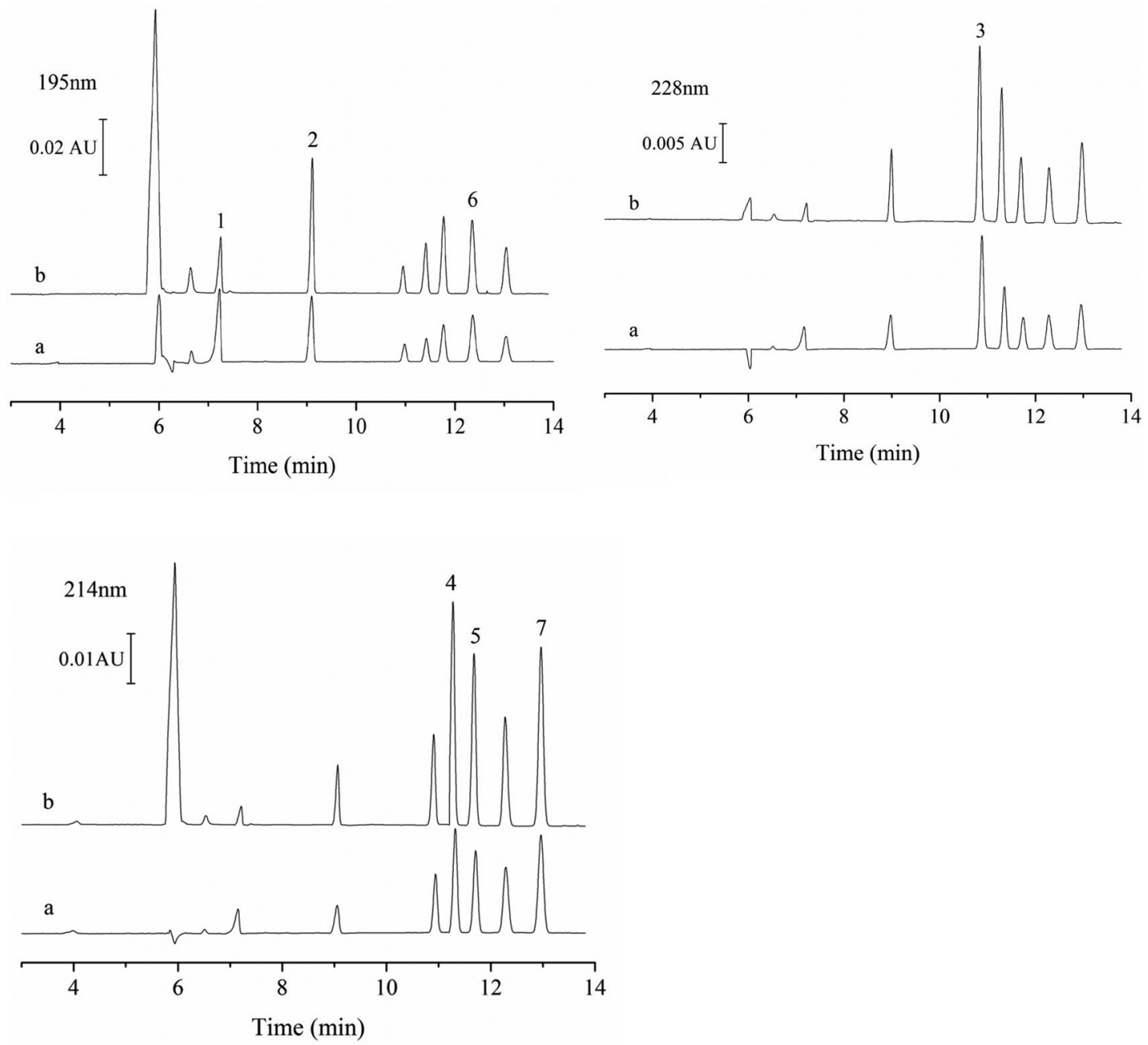

Figure 3. Electropherograms of seven phenols standard solution monitored at 195, 214 and $228 \mathrm{~nm}$ with the concentration of (a) $20 \mu \mathrm{g} / \mathrm{mL}$ and (b) $50 \mu \mathrm{g} / \mathrm{L}$ after MIPs-DSPE. Peak identification: (1) phenol; (2) 4-CP; (3) PCP; (4) 2,4,6-TCP; (5) 2,4-DCP; (6) 2-CP; (7) 2,6-DCP. CE conditions: $15 \mathrm{mmol} / \mathrm{L} \mathrm{Na2B} 4 \mathrm{O} 7 \cdot \mathrm{H} 2 \mathrm{O}$ containing $15 \%(\mathrm{v} / \mathrm{v})$ methanol at $\mathrm{pH}=9.8$, injection $5 \mathrm{~s}$ with $0.5 \mathrm{psi}$, $+15 \mathrm{kV}$ applied voltage.

Table 1. Analytical parameters of MIPs-DSPE-CE method for the determination of seven phenols

\begin{tabular}{|c|c|c|c|c|c|c|c|c|}
\hline Compounds & Calibration curves ${ }^{\text {a) }}$ & $\begin{array}{l}\text { Correlation } \\
\text { coefficient }\left(R^{2}\right)\end{array}$ & $\begin{array}{l}\text { Linear range } \\
(\mu \mathrm{g} / \mathrm{L})\end{array}$ & $\begin{array}{l}\mathrm{LOD} \\
(\mu \mathrm{g} / \mathrm{L})\end{array}$ & $\begin{array}{l}\mathrm{LOO} \\
(\mu \mathrm{g} / \mathrm{L})\end{array}$ & $\begin{array}{l}\text { Intraday precision }{ }^{\text {b) }} \\
(\mathrm{RSD}, \%)(n=5)\end{array}$ & $\begin{array}{l}\text { Interday precision }{ }^{\text {b) }} \\
(\text { RSD, \%) }(n=5)\end{array}$ & $\begin{array}{l}\text { Enrichment } \\
\text { factors }\end{array}$ \\
\hline Phenol & $y=584.1 x+472.5$ & 0.9959 & $2-200$ & 0.44 & 1.45 & 6.04 & 6.79 & 70 \\
\hline 4-CP & $y=1569 x+84.86$ & 0.9938 & $2-300$ & 0.19 & 0.63 & 2.87 & 3.11 & 198 \\
\hline PCP & $y=1042 x+60.05$ & 0.9968 & $1.5-150$ & 0.32 & 1.05 & 3.83 & 6.06 & 234 \\
\hline 2,4,6-ТCP & $y=1608 x+159.2$ & 0.9970 & $2-400$ & 0.18 & 0.59 & 1.91 & 2.02 & 242 \\
\hline 2,4-DCP & $y=1313 x+195.9$ & 0.9989 & $2-200$ & 0.25 & 0.83 & 3.22 & 4.60 & 239 \\
\hline 2-CP & $y=1069 x+178.3$ & 0.9971 & $2-300$ & 0.35 & 1.16 & 4.22 & 5.08 & 168 \\
\hline 2,6-DCP & $y=1591 x+462.6$ & 0.9985 & $2-200$ & 0.31 & 1.02 & 4.12 & 4.58 & 209 \\
\hline
\end{tabular}

a) $y$ and $x$ stand for the peak area and the concentration $(\mu \mathrm{g} / \mathrm{L})$ of all the analytes, respectively.

b) Concentration of $100 \mu \mathrm{g} / \mathrm{L}$ individual for all the analytes was used for the assays of intraday and interday precision.

preparation procedure of precipitation polymerization, high capacity and fast adsorption, simultaneous separation of seven phenols in a short time. Therefore, the developed method indicated great potential for effective trace analysis of phenols in real water samples.

\subsection{Applications in real samples}

The feasibility and applicability of the developed method was assessed by the determination of seven phenols in lake water, tap water, seawater and tannery wastewater samples. The 
electropherograms of four real water samples at the wavelength of 195, 214 and $228 \mathrm{~nm}$ were shown in Supporting Information Fig. S4. As seen, the endogenous target analytes were not detected in the four water samples.

To further evaluate the method performance in complex matrices, the recoveries were investigated by spiking seven phenols at three levels $(10,50$ and $100 \mu \mathrm{g} / \mathrm{L})$ and analyzing four replicates for each concentration. The results were listed in Supporting Information Table S2. Recoveries were in the range of $77.86-104.3 \%$ for lake water, $81.21-96.39 \%$ for tap water, $77.71-97.76 \%$ for seawater and $70.75-106.7 \%$ for tannery wastewater, with the RSD ranging from $1.20-5.42 \%$ for lake water, $1.27-5.61 \%$ for tap water, $1.28-5.96 \%$ for seawater and $1.15-6.28 \%$ for tannery wastewater. These results demonstrated that the developed MIPs-DSPE-CE method was practically applicable for the determination of trace phenols in water samples.

\section{Concluding remarks}

In this study, MIPs were prepared by precipitation polymerization using 2,4,6-TCP as a template, MAA as a functional monomer and EGDMA as a cross-linker, and were employed as DSPE sorbents coupled with CE-DAD for the simultaneous enrichment and determination of seven phenolic compounds in aqueous samples. For MIPs-DSPE, smaller amount of polymers and faster adsorption equilibrium were achieved compared with conventional SPE. CE separation needs shorter time which can simultaneously separate seven phenols within $13.5 \mathrm{~min}$ and obtains satisfactory detection sensitivity higher than or comparable to HPLC and GC. Under the optimized conditions, the presented method was applied in real water samples and showed good precision and accuracy with recoveries ranging from 70.75 to $106.7 \%$ and RSDs from 1.15 to $6.28 \%$. Given the advantages, further research should focus on the development of molecular imprinting technology to improve the performances of MIPs, and thereby MIPs related highly efficient, simple and reliable offline pretreatment techniques and/or online pretreatment techniques.

This work was financially supported by the Strategic Priority Research Program of the Chinese Academy of Sciences (XDA11020702), the National Natural Science Foundation of China (21477160, 21275158), and the National 863 Plan of China (2007AA092001-5).

The authors have declared no conflict of interest.

\section{References}

[1] Bayramoğlu, G., Arıca, M. Y., J. Hazard. Mater. 2008, 156, 148-155.

[2] Rodríguez, I., Llompart, M. P., Cela, R., J. Chromatogr. A 2000, 885, 291-304.

[3] Gimeno, O., Carbajo, M., Beltrán, F. J., Rivas, F. J., J. Hazard. Mater. 2005, 119, 99-108.
[4] Padilla-Sanchez, J. A., Plaza-Bolanos, P., RomeroGonzalez, R., Barco-Bonilla, N., Martinez-Vidal, J. L., Garrido-Frenich, A., Talanta 2011, 85, 2397-2404.

[5] Faludi, T., Balogh, C., Serfozo, Z., Molnar-Perl, I., Environ. Sci. Pollut. Res. 2015, 22, 11966-11974.

[6] Liu, J. F., Liang, X., Chi, Y. G., Jiang, G. B., Cai, Y. Q., Zhou, O. X., Liu, G. G., Anal. Chim. Acta 2003, 487, 129-135.

[7] Blanco, E., Casais, M. C., Mejuto, M. C., Cela, R., J. Chromatogr. A 2005, 1068, 189-199.

[8] Naeeni, M. H., Yamini, Y., Rezaee, M., Seidi, S., J. Sep. Sci. 2012, 35, 2469-2475.

[9] Peng, J. F., Liu, J. F., Hu, X. L., Jiang, G. B., J. Chromatogr. A 2007, 1139, 165-170.

[10] Zhou, L. F., He, X. G., Qiao, J.Q., Lian, H. Z., Ge, X., Chen, H. Y., J. Chromatogr. A 2012, 1256, 15-21.

[11] Suliman, F. E. O., Al-Kindi, S. S., Al-Kindy, S. M. Z., Al-Lawati, H. A. J., J. Chromatogr. A 2006, 1101, 179-184.

[12] Su, F., Zhang, P., J. Sep. Sci. 2011, 34, 495-499.

[13] Park, S. T., Kim, J., Choi, K., Lee, H. R., Chung, D. S., Electrophoresis 2012, 33, 2961-2968.

[14] Wang, Q., Qiu, H., Li, J., Liu, X., Jiang, S., Electrophoresis 2010, 1217, 5434-5439.

[15] Moradi, M., Yamini, Y., Esrafili, A., Seidi, S., Talanta 2010, 82, 1864-1869.

[16] Liu, X., Yin, J., Zhu, L., Zhao, G., Zhang, H., Talanta 2011, $85,2451-2457$.

[17] Kim, D., Han, J., Choi, Y., Anal. Bioanal. Chem. 2013, 405, 377-387.

[18] Liu, L., Liu, H. X., Li, Y., Wang, X. M., Du, X. Z., Anal. Lett. 2014, 47, 2508-2521.

[19] Li, Y., Li, W., Wang, Y., Zhou, H., Hu, G., Zhang, N., Sun, C., J. Sep. Sci. 2013, 36, 2121-2127.

[20] Hu, C., Chen, B., He, M., Hu, B., J. Chromatogr. A 2013, $1300,165-172$

[21] He, H., Liu, S., Meng, Z., Hu, S., J. Chromatogr. A 2014, 1361, 291-298.

[22] Sharma, N., Jain, A., Singh, V. K., Verma, K. K., Talanta 2011, 83, 994-999.

[23] Abolghasemi, M. M., Yousefi, V., J. Chromatogr. A 2014, 1345, 9-16.

[24] Andersson, L. I., Hardenborg, E., Sandberg-Ställ, M., Möller, K., Henriksson, J., Bramsby-Sjöström, I., Olsson, L. I., Abdel-Rehim, M., Anal. Chim. Acta 2004, 526, 147-154.

[25] Wen, Y. Y., Chen, L., Li, J. H., Liu, D.Y., Chen, L. X., TrAC Trend. Anal. Chem. 2014, 59, 26-41.

[26] Chen, L. X., Wang, X. Y., Lu, W. H., Wu, X. Q., Li, J. H., Chem. Soc. Rev. 2016, 45, 2137-2211.

[27] Sun, X., Wang, J., Li, Y., Jin, J., Yang, J., Li, F., Shah, S. M., Chen, J., J. Chromatogr. A 2014, 1360, 9-16.

[28] Xu, S. F., Chen, L. X., Li, J. H., Qin, W., Ma, J. P., J. Mater. Chem. 2011, 21, 12047-12053.

[29] Sun, H., Lai, J. P., Chen, F., Zhu, D. R., Anal. Bioanal. Chem. 2015, 407, 1745-1752.

[30] Feng, Q., Zhao, L., Lin, J. M., Anal. Chim. Acta 2009, 650, 70-76. 
[31] Feng, Q. Z., Zhao, L. X., Yan, W., Lin, J. M., Zheng, Z. X., J. Hazard. Mater. 2009, 167, 282-288.

[32] Qi, S., Zhang, H., Zhu, Q., Chen, H., Dong, Y., Zhou, L., Ren, C., Chen, X., Anal. Methods 2014, 6, 1219-1226.

[33] Anastassiades, M., Lehotay, S. J., tajnbaher, D., Schenck, F. J., J. AOAC Int. 2003, 86, 412-431.

[34] Yoshimatsu, K., Reimhult, K., Krozer, A., Mosbach, K., Sode, K., Ye, L., Anal. Chim. Acta 2007, 584, 112-121.

[35] Yang, M., Han, A., Duan, J., Li, Z., Lai, Y., Zhan, J., J. Hazard. Mater. 2012, 238, 63-70.
[36] Shen, H. Y., Chen, Z. X., Li, Z. H., Hu, M. Q., Dong, X. Y., Xia, Q. H., Colloids Surface A 2015, 481, 439-450.

[37] Baggiani, C., Anfossi, L., Giovannoli, C., Tozzi, C., J. Chromatogr. B 2004, 804, 31-41.

[38] Hu, Y., Liu, R., Li, Y., Li, G., J. Sep. Sci. 2010, 33, 20172025.

[39] Surikumaran, H., Mohamad, S., Muhamad Sarih, N., Muggundha Raoov, R., Sep. Sci. Technol. 2015, 50, 23422351. 